\title{
A Generalized Sampling Method for Finite-Rate-of-Innovation-Signal Reconstruction
}

\author{
Chandra Sekhar Seelamantula, Member, IEEE, and Michael Unser, Fellow, IEEE
}

\begin{abstract}
The problem of sampling signals that are not admissible within the classical Shannon framework has received much attention in the recent past. Typically, these signals have a parametric representation with a finite number of degrees of freedom per time unit. It was shown that, by choosing suitable sampling kernels, the parameters can be computed by employing high-resolution spectral estimation techniques. In this letter, we propose a simple acquisition and reconstruction method within the framework of multichannel sampling. In the proposed approach, an infinite stream of nonuniformly-spaced Dirac impulses can be sampled and accurately reconstructed provided that there is at most one Dirac impulse per sampling period. The reconstruction algorithm has a low computational complexity, and the parameters are computed on the fly. The processing delay is minimal-just the sampling period. We propose sampling circuits using inexpensive passive devices such as resistors and capacitors. We also show how the approach can be extended to sample piecewise-constant signals with a minimal change in the system configuration. We provide some simulation results to confirm the theoretical findings.
\end{abstract}

Index Terms - Exponential splines, finite rate of innovation, generalized sampling, localizing filter, piecewise-constant functions, streams of Dirac impulses.

\section{INTRODUCTION}

$\mathbf{R}$ ECENTLY, there has been a lot of interest in sampling and reconstructing signals that cannot be handled within the classical framework of Shannon's sampling theory. Examples of such signals include streams of Dirac impulses, and various types of nonuniform splines such as piecewise-polynomial, piecewise-exponential, and piecewise-harmonic functions. These signals are uniquely specified by a discrete set of parameters (for example, the amplitudes and positions of the singularities) and are said to possess a finite rate of innovation (FRI) [1]. Potential applications of FRI sampling are in high-rate distributed analog-to-digital conversion, image processing, digital processing of neuronal signals, bioimaging, analysis of stochastic point-processes, ultrawideband communication, etc.

The notion of FRI sampling was first introduced by Vetterli et al. [1]. They formulated the reconstruction problem as one of parameter estimation and established a link to the annihilating filter approach, which forms the main ingredient of many super-resolution spectral estimation techniques. In fact, the FRI problem involving a periodic stream of Dirac impulses

Manuscript received July 09, 2008; revised August 13, 2008. This work was supported by the Swiss National Science Foundation (SNSF) under Grant 200020-101821. The associate editor coordinating the review of this manuscript and approving it for publication was Prof. Yimin Zhang.

The authors are with the Biomedical Imaging Group, École polytechnique fédérale de Lausanne (EPFL), Lausanne, Switzerland (e-mail: chandrasekhar. seelamantula@epfl.ch; michael.unser@epfl.ch).

Color versions of one or more of the figures in this paper are available online at http://ieeexplore.ieee.org.

Digital Object Identifier 10.1109/LSP.2008.2006316 addressed in [1] is exactly the dual of the classical harmonic-retrieval problem discussed in [2]. Vetterli et al. considered two types of kernels: 1) infinitely-supported functions such as the Gaussian and the sinc; and 2) compactly-supported ones such as B-splines [3], which overcome certain drawbacks of the first type and give rise to stable estimation techniques. Dragotti et al. [4] introduced spline-based algorithms for recovering FRI signals from their moments. Thereby, they showed that the classical annihilating filter approach can be extended to solve equations involving power-sum series.

The inspiration for the present work lies in the notion of generalized or multichannel sampling first proposed by Papoulis in the context of bandlimited signals [5]. Subsequently, Unser and Zerubia extended the theory to the class of square-integrable functions and performed optimal reconstruction in shift-invariant spaces [6]. The development of multichannel approaches for FRI signals has been initiated only recently [7], [8]. Kusuma and Goyal proposed new sampling approaches using a bank of integrators and B-splines. Their scheme has a successive approximation property, which is useful for reconstructing an unknown number of impulses over a finite interval of time and for detecting suboptimal modeling [7]. Baboulaz and Dragotti employed a distributed setup for acquiring FRI signals and demonstrated applications to image registration based on continuous image moments. These results were then used for performing super-resolution image restoration within the framework of Wiener filtering [8].

In this letter, we propose a simple two-channel method for sampling FRI signals. We first focus on sampling a stream of Dirac impulses for it is the canonical FRI signal. We maintain the same overall sampling rate as the standard approaches and propose a low-complexity reconstruction technique. We then show how the approach can be extended to sample piecewiseconstant functions. We provide some simulation results to support the theoretical analysis. In our analysis, we assume that the measurements are noise-free.

\section{FROM DiRAC IMPULSES TO KRONECKER IMPULSES}

Consider the impulse train

$$
x(t)=\sum_{\ell=1}^{L} a_{\ell} \delta_{D}\left(t-t_{\ell}\right)
$$

where $\delta_{D}(\cdot)$ denotes the Dirac impulse. The impulses are assumed to follow a natural time-ordering; i.e., $t_{1}<t_{2}<$ $\cdots<t_{L-1}<t_{L}$. The objective is to compute the parameters $\left\{a_{\ell}, t_{\ell} ; t_{\ell} \in \mathbb{R}, a_{\ell} \in \mathbb{C} ; 1 \leq \ell \leq L\right\}$ based on some measurements of $x(t)$.

Impulses are best characterized in a distributional framework, and their properties are revealed through their action on a set of analysis functions. From a signal-processing perspective, linear shift-invariant systems can be viewed as operators that generate 


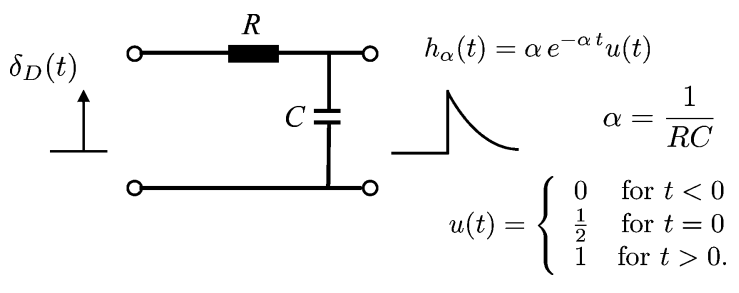

Fig. 1. First-order resistor-capacitor network in lowpass configuration. The system parameter $\alpha$ is the reciprocal of the time-constant.

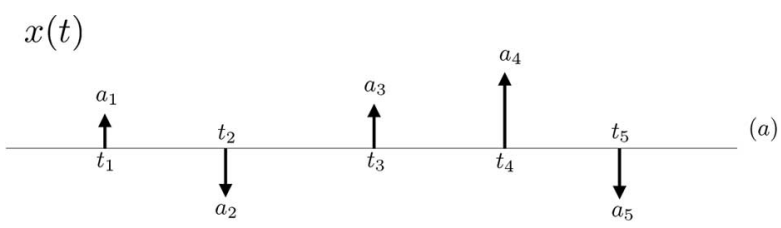

$y_{\alpha}(t)$

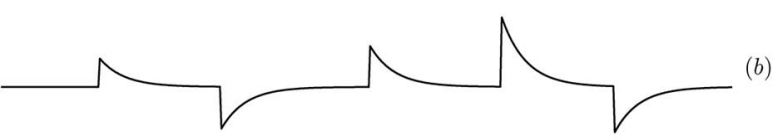

$y_{\alpha}(n T)$

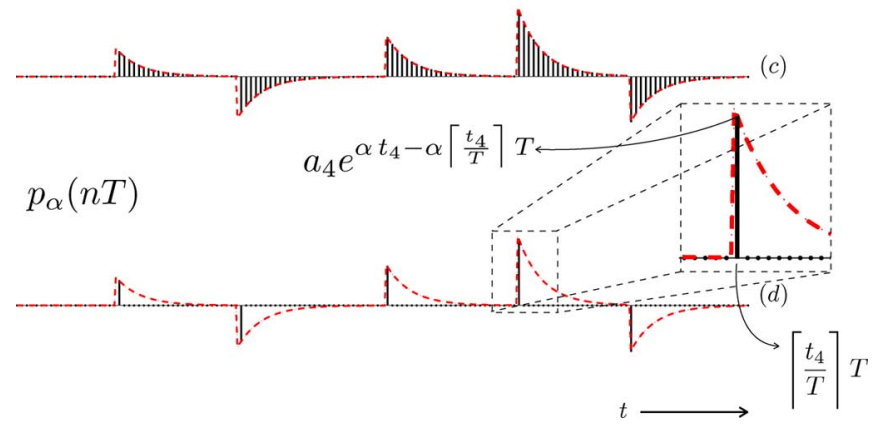

Fig. 2. From Dirac impulses to Kronecker impulses. The subplots (a)-(d) correspond to (1)-(4), respectively. The zoomed plot in (d) illustrates the upwardrounding effect.

analysis functions and are specified by their impulse responses. The most commonly encountered practical systems are characterized by linear differential equations or rational transfer functions. Let us consider the passive first-order resistor-capacitor (RC) network in lowpass configuration [cf. Fig. 1] specified by the impulse response $h_{\alpha}(t)=\alpha e^{-\alpha t} u(t)$, where $u(t)$ is the unit-step function. When excited with $x(t)$ given in (1), the system would yield the following response:

$$
y_{\alpha}(t)=\left(x * h_{\alpha}\right)(t)=\alpha \sum_{\ell=1}^{L} a_{\ell} e^{-\alpha\left(t-t_{\ell}\right)} u\left(t-t_{\ell}\right)
$$

where the convolution must be understood in the sense of distributions. In Fig. 2(a) and (b), we show examples of $x(t)$ and $y_{\alpha}(t)$, respectively. If $y_{\alpha}(t)$ is sampled every $T$ seconds, it gives rise to the discrete-time sequence [cf. Fig. 2(c)]

$$
y_{\alpha}(n T)=\alpha \sum_{\ell=1}^{L} a_{\ell} e^{-\alpha\left(n T-t_{\ell}\right)} u\left(n T-t_{\ell}\right), n \in \mathbb{Z} .
$$

Next, consider a discrete-time finite-impulse-response filter specified by the $Z$-transform: $G_{\alpha}(z)=$ $(1 / \alpha)\left(1-e^{-\alpha T} z^{-T}\right)$. Note that $G_{\alpha}(z)$ is the convolutional-inverse of the discrete-time exponential $\alpha e^{-\alpha n T} u(n T)$.

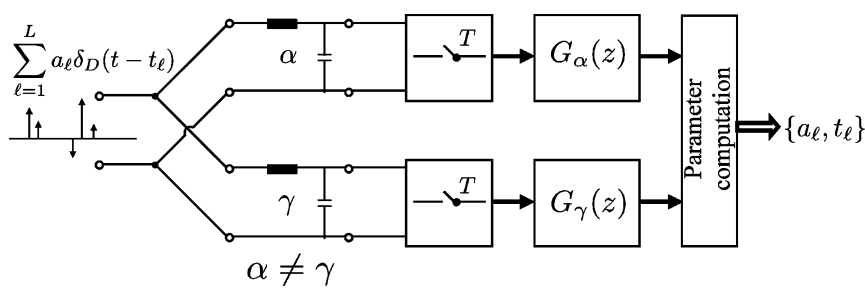

Fig. 3. Two-channel sampling of a stream of Dirac impulses by using first-order $\mathrm{RC}$ networks. The parameter computation is given in (6)-(9).

Therefore, when the sequence in (3) is processed by $G_{\alpha}(z)$, it gives rise to a stream of Kronecker impulses [cf. Fig. 2(d)] as shown in the following:

$$
\begin{aligned}
p_{\alpha}(n T) & =\sum_{\ell=1}^{L} a_{\ell} e^{-\alpha n T+\alpha t_{\ell}}\left(u\left(n T-t_{\ell}\right)-u\left((n-1) T-t_{\ell}\right)\right) \\
& =\sum_{\ell=1}^{L} a_{\ell} e^{-\alpha r\left(t_{\ell}\right) T+\alpha t_{\ell}} \delta_{K}\left[n-r\left(t_{\ell}\right)\right]
\end{aligned}
$$

where $\delta_{K}[\cdot]$ denotes the Kronecker impulse and $r\left(t_{\ell}\right)=$ $\left\lceil t_{\ell} / T\right\rceil$, with $\lceil\cdot\rceil$ indicating the ceiling operation. The positions of the Kronecker impulses are equal to the positions of the Dirac impulses rounded upwards to lie on the sampling grid. The amplitude of the Kronecker impulse carries information about the position as well as the amplitude of the corresponding Dirac impulse in a separable fashion. From Fig. 2(d), it is clear that the upward-rounding operation places a limit on resolvability; i.e., Dirac impulses that are closer apart than $T$ give rise to overlapping Kronecker impulses and hence cannot be resolved directly. This issue does not arise if there is at most one Dirac impulse in a sampling interval; i.e., if

$$
\min _{2 \leq \ell \leq L}\left\{t_{\ell}-t_{\ell-1}\right\}>T \text {. }
$$

Note that the above condition is similar to the one stated in [1] for sampling bilevel signals by employing a linear B-spline kernel. In some cases, the underlying mechanics of signal generation places a lower limit on the spacing between two consecutive events, and may even be available $a$ priori, thereby enabling an appropriate choice of $T$.

\section{Two-Channel SAmpling OF A DiRAC IMPUlSE Train}

In the foregoing analysis, we have seen that the amplitudes of the Kronecker impulses are separable functions of the amplitudes and positions of the Dirac impulses. This property gives rise to a simple and efficient reconstruction technique as we shall show next. To proceed further, we consider another set of measurements obtained by employing a different sampling kernel. In principle, the second set of acquisitions may be made by using a totally different filter; in the present development, we use a similar RC network as shown in Fig. 1, but with a different parameter $\gamma$ [cf. Fig. 3]. We shall soon see the advantages this choice of filters has to offer.

Corresponding to the network with parameter $\gamma$, we have the sequence $p_{\gamma}(n T)$, which is obtained from (4) by replacing $\alpha$ with $\gamma$. The $\ell$ th nonzero values in $p_{\alpha}(n T)$ and $p_{\gamma}(n T)$ occur at the time instant $r\left(t_{\ell}\right) T=\left\lceil t_{\ell} / T\right\rceil T$, just after the $\ell$ th Dirac impulse has excited the respective analog systems. From the nonzero values, we compute two new quantities as follows: 


$$
\begin{aligned}
& q_{\alpha}[\ell]=p_{\alpha}\left(r\left(t_{\ell}\right) T\right) e^{\alpha r\left(t_{\ell}\right) T}=a_{\ell} e^{\alpha t_{\ell}}, \text { and } \\
& q_{\gamma}[\ell]=p_{\gamma}\left(r\left(t_{\ell}\right) T\right) e^{\gamma r\left(t_{\ell}\right) T}=a_{\ell} e^{\gamma t_{\ell}} .
\end{aligned}
$$

Solving (6) and (7) for $t_{\ell}$ and $a_{\ell}$, we get that

$$
\begin{aligned}
t_{\ell} & =\frac{1}{\alpha-\gamma} \ln \left(\frac{q_{\alpha}[\ell]}{q_{\gamma}[\ell]}\right), \text { and } \\
a_{\ell} & =\exp \left(-\frac{\alpha}{\alpha-\gamma} \ln \left(\frac{q_{\alpha}[\ell]}{q_{\gamma}[\ell]}\right)\right) q_{\alpha}[\ell] .
\end{aligned}
$$

Equations (6)-(9) thus constitute a closed-form solution for the nonlinear problem of computing the parameters of $x(t)$. The delay after the $\ell$ th impulse has excited the systems and before $\left\{a_{\ell}, t_{\ell}\right\}$ can be computed is $T$. The reconstruction technique operates locally; therefore, it can be applied to an infinitely long impulse train. The special case where an impulse is located on the sampling grid, i.e., when $t_{\ell}$ is an integer multiple of $T$, is indicated by the unique condition: $p_{\alpha}\left(r\left(t_{\ell}\right) T\right)=p_{\gamma}\left(r\left(t_{\ell}\right) T\right)=$ $a_{\ell} / 2, p_{\alpha}\left(r\left(t_{\ell}\right) T+T\right)=\left(a_{\ell} / 2\right) e^{-\alpha T}$, and $p_{\gamma}\left(r\left(t_{\ell}\right) T+T\right)=$ $\left(a_{\ell} / 2\right) e^{-\gamma T}$. In this case, we have that $t_{\ell}=r\left(t_{\ell}\right) T$ and $a_{\ell}=$ $2 p_{\alpha}\left(r\left(t_{\ell}\right) T\right)$.

We summarize our results in the form of a proposition.

Proposition 1: The stream of Dirac impulses $x(t)=$ $\sum_{\ell \in \mathbb{Z}} a_{\ell} \delta_{D}\left(t-t_{\ell}\right)$ is uniquely specified by the samples $y_{\alpha}(n T)=\left(x * h_{\alpha}\right)(n T)$ and $y_{\gamma}(n T)=\left(x * h_{\gamma}\right)(n T), n \in \mathbb{Z}$, where $h_{\alpha}(t)=\alpha e^{-\alpha t} u(t), h_{\gamma}(t)=\gamma e^{-\gamma t} u(t)$, and $\alpha \neq \gamma$, provided that there is at most one impulse in each sampling interval.

\section{SPLINE EQUIVALENCE}

The causal exponential $\alpha e^{-\alpha t} u(t)$ and the filter $G_{\alpha}(z)=$ $(1 / \alpha)\left(1-e^{-\alpha T} z^{-T}\right)$ are sufficient indicators to hint that the foregoing analysis can be recast within the framework of splines, in particular cardinal E-splines [9], which are of the exponential variety. The filter $G_{\alpha}(z)$ is immediately identified as the exponential-localizing filter. Note that $p_{\alpha}(n T)$ given in (4) is the weighted finite difference

$$
\begin{aligned}
p_{\alpha}(n T) & =\left(y_{\alpha}(n T)-e^{-\alpha T} y_{\alpha}((n-1) T)\right) / \alpha \\
& =\left(x * \beta_{\alpha}\right)(n T)
\end{aligned}
$$

where $\beta_{\alpha}(t)=(1 / \alpha)\left(h_{\alpha}(t)-e^{-\alpha T} h_{\alpha}(t-T)\right)$ is the firstorder E-spline [9] with parameter $\alpha$, at scale $T$. Similarly, it can be shown that $p_{\gamma}(n T)=\left(x * \beta_{\gamma}\right)(n T)$. Thus, the process of filtering, sampling, and localizing described in Section II is equivalent to sampling with an E-spline kernel.

If the E-splines are scaled versions of one another, then we have reconstruction from measurements taken at two scales. An interesting scenario occurs when one of the E-splines is replaced by a causal polynomial B-spline of order zero; i.e., either $\alpha=0$ or $\gamma=0$. The B-spline channel output would then straightaway give the amplitudes of the Dirac impulses. The positions of the impulses can be computed from the samples of the E-spline channel. Thus, we can have a hybrid spline-based sampling system, which further simplifies the calculations.

\section{TWO-CHANNEL SAMPLING OF PIECEWISE-CONSTANT SigNALS}

We next show that, by an appropriate choice of the sampling circuit, one can sample and reconstruct piecewise-constant signals. Consider the function

$$
g(t)=\sum_{\ell=1}^{L} b_{\ell}\left[u\left(t-t_{\ell}\right)-u\left(t-t_{\ell-1}\right)\right], \text { for } t>0
$$

and zero elsewhere; $t_{0}=0$ and $\left\{t_{\ell} ; \ell=1,2, \ldots, L\right\}, 0<t_{1}<$ $t_{2}<\cdots<t_{L}$, are the instants at which $g(t)$ has discontinuities. Consider the distributional derivative of $g(t)$ given by

$$
\frac{\mathrm{d} g(t)}{\mathrm{d} t}=\sum_{\ell=1}^{L} a_{\ell} \delta_{D}\left(t-t_{\ell}\right)
$$

where $a_{\ell}=b_{\ell}-b_{\ell-1}$, for $\ell=1,2, \ldots, L$, and $b_{0}=0$. The signal in (12) is identical to that in (1), and therefore, the parameters $\left\{a_{\ell}, t_{\ell}\right\}$ can be computed by employing the method described in Sections II and III. The amplitudes $\left\{b_{\ell} ; \ell=1,2, \ldots, L\right\}$ can then be computed recursively as $b_{\ell}=b_{\ell-1}+a_{\ell}$ together with the initial condition $b_{0}=0$.

The sampling scheme proposed above can be implemented by employing RC networks arranged in highpass configuration; i.e., the output voltage is measured across the resistor instead of measuring across the capacitor. This claim can be easily verified by working in the Fourier domain. Consider the following factorization of the frequency response of the RC highpass network:

$$
\widehat{h}_{\alpha}^{h p}(\omega)=\mathrm{j} \omega \cdot \frac{1}{\mathrm{j} \omega+\alpha} .
$$

The $\mathrm{j} \omega$ factor corresponds to the ideal differentiator and $(1 /(\mathrm{j} \omega+\alpha))$ is the frequency response of the RC lowpass network (up to the scale factor $\alpha$ ) used in Section II. Thus, it turns out that the first-order RC network can be used to filter streams of Dirac impulses as well as piecewise-constant signals depending on whether it is configured as lowpass or highpass, respectively.

\section{SiMULATIONS}

To support our theoretical findings, we report some simulation results. Consider a stream of six Dirac impulses with randomly chosen amplitudes and positions such that in each sampling interval $(T=0.005 \mathrm{~s})$, there is at most one impulse [cf. Fig. 4(a)]. The impulses are analyzed by using two RC networks with parameters $\alpha=3$ and $\gamma=2.1$. These values are chosen for the sake of illustration. The reconstructed impulses are shown in Fig. 4(b). The reconstruction is exact to numerical precision. Similar results were obtained for other choices of $\alpha$ and $\gamma$. Next, consider the piecewise-constant signal shown in Fig. 4(c), where the transition points are farther apart than $T$ and where the amplitudes are assigned randomly. The sampling setup is the same as the one described above. The reconstructed signal is shown in Fig. 4(d); in this case also, accurate reconstruction is achieved.

\section{CONCLUSIONS}

We have addressed the canonical problem of sampling and reconstructing a stream of Dirac impulses. We have proposed a two-channel sampling approach to retrieve the parameters of the impulses using simple computations. In principle, the technique 

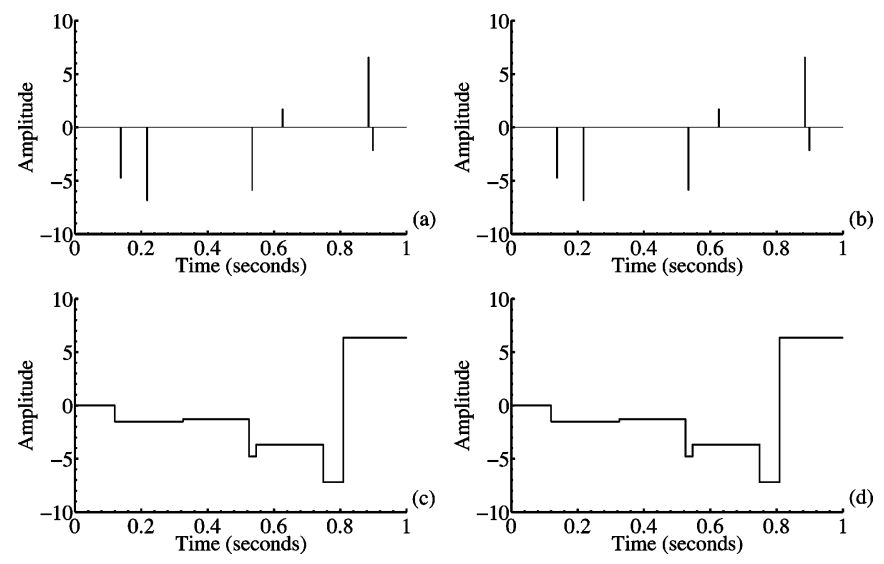

Fig. 4. (a) Stream of six Dirac impulses (ground truth) and (b) Reconstruction; (c) Piecewise-constant signal (ground truth) and (d) Reconstruction.

TABLE I

COMPARISON OF THE STANDARD AND THE PROPOSED APPROACHES

\begin{tabular}{|c|c|c|}
\hline Feature & Standard approaches & Proposed method \\
\hline $\begin{array}{l}\text { Prior knowledge of } \\
\text { number of impulses }\end{array}$ & $\begin{array}{l}\text { Maximum number of } \\
\text { impulses }\end{array}$ & None \\
\hline Inter-impulse spacing & No constraint & Must be greater than $T$ \\
\hline Super-resolution & Yes & $\begin{array}{l}\text { Yes, but only if (5) is } \\
\text { satisfied }\end{array}$ \\
\hline Hardware complexity & $\begin{array}{l}\text { Specific kernel; single } \\
\text { sampling circuit }\end{array}$ & $\begin{array}{l}\text { Simple RC networks } \\
\text { two sampling circuit } \\
\text { (each operating at hal } \\
\text { the total sampling rate) }\end{array}$ \\
\hline Estimation algorithm & Sophisticated & Straightforward \\
\hline Processing & $\begin{array}{l}\text { Block-wise (large de- } \\
\text { lay) }\end{array}$ & Online (small delay) \\
\hline $\begin{array}{l}\text { Local rate of innova- } \\
\text { tion }(\Gamma)\end{array}$ & $\begin{array}{l}\Gamma_{2 L S T} \leq \frac{2}{S T} ; S \text { is } \\
\text { the support of the B- } \\
\text { spline sampling ker- } \\
\text { nel; } S \geq 2 \text { (cf. The- } \\
\text { orem } 2,[4]) ; 2 L S T \text { is } \\
\text { the size of the interval }\end{array}$ & $\begin{array}{l}\Gamma_{2 L S T} \leq \frac{2}{T} ; \text { Choice } \\
\text { of kernel dependent on } \\
\text { the rate of innovation } \\
\text { (cf. (5) and (10)) }\end{array}$ \\
\hline
\end{tabular}

is applicable to an infinitely long impulse train, without having to process it block-wise. A comparison of the salient features of the proposed method with the standard FRI approaches [1], [4] is given in Table I. The overall sampling rate in the two approaches is the same. The proposed method has a number of advantageous features, but it has the fundamental limitation that it cannot resolve impulses that are closer apart than $T$, which is not the case for the previously published techniques [1], [4].

We would like to emphasize that the filtering interpretation employing RC networks does not restrict the analysis to positive $\alpha$ and $\gamma$. One could, for instance, also choose complex exponentials such as the causal Fourier bases (imaginary $\alpha, \gamma$; appropriately chosen), or their exponentially-damped versions (complex-valued $\alpha, \gamma)$. In this letter, we have presented a proof of concept and some generalizations are possible-multiple channels, higher-order sampling kernels, etc., which are perhaps desirable in the presence of noise. A generalization to secondorder E-spline sampling kernels is given in the Appendix .

We have also demonstrated the applicability of the proposed sampling method to piecewise-constant signals. Further extensions to piecewise-polynomial signals or nonuniform splines can be done by following the ideas proposed in [4]. In principle, one may also derive robust subspace-type techniques within the framework of multichannel sampling-we believe that this is a research topic in its own right.

\section{APPENDIX}

Consider sampling $x(t)$ in (1) with two second-order E-spline kernels $\beta_{\vec{\alpha}}(t)$ and $\beta_{\vec{\gamma}}(t)$, where $\vec{\alpha}=\left\{\alpha_{1}, \alpha_{2}\right\}$ and $\vec{\gamma}=\left\{\gamma_{1}, \gamma_{2}\right\}$, both at scale $T$. Assume that the kernels differ in at least one of the parameters. If two consecutive impulses are farther apart than $2 T$ (the support of the kernels), and the sampling period is $2 T$, then, corresponding to each impulse, we have a nonzero sample in each channel. For example, consider the following samples corresponding to the $\ell$ th impulse:

$$
\begin{aligned}
& q_{\alpha}[\ell]=a_{\ell} c_{\alpha}\left(e^{-\alpha_{2} \Delta_{\ell}}-e^{-\alpha_{1} \Delta_{\ell}}\right) \text { and } \\
& q_{\gamma}[\ell]=a_{\ell} c_{\gamma}\left(e^{-\gamma_{2} \Delta_{\ell}}-e^{-\gamma_{1} \Delta_{\ell}}\right)
\end{aligned}
$$

where $\Delta_{\ell}=r\left(t_{\ell}\right) T-t_{\ell}, c_{\alpha}=1 /\left(\alpha_{1}-\alpha_{2}\right)$, and $c_{\gamma}=$ $1 /\left(\gamma_{1}-\gamma_{2}\right)$. Dividing (14) by (15) and rearranging gives an equation in terms of $t_{\ell}$, which can be solved for in closed-form only for specific values of the parameters of the E-splines. For example, if we choose $\alpha_{1}=0, \alpha_{2}=2, \gamma_{1}=1$, and $\gamma_{2}=2$, we get a quadratic equation in $e^{t_{\ell}}$, the positive root of which then corresponds to the solution of $t_{\ell}$. In the general case, iterative solvers may be required. Substituting the value of $t_{\ell}$ thus computed in either (14) or (15) yields $a_{\ell}$ in a straightforward fashion. Thus, the procedure can also be extended to second-order kernels. The main advantage of working with first-order kernels, however, is the simplicity of the reconstruction technique.

\section{REFERENCES}

[1] M. Vetterli, P. Marziliano, and T. Blu, "Sampling signals with finite rate of innovation," IEEE Trans. Signal Process., vol. 50, no. 6, pp. 1417-1428, Jun. 2002.

[2] P. Stoica and R. Moses, Introduction to Spectral Analysis. Englewood Cliffs, NJ: Prentice-Hall, 2000.

[3] M. Unser, "Splines: A perfect fit for signal and image processing," IEEE Signal Process. Mag., vol. 16, no. 6, pp. 22-38, Nov. 1999.

[4] P. L. Dragotti, M. Vetterli, and T. Blu, "Sampling moments and reconstructing signals of finite rate of innovation: Shannon meets StrangFix," IEEE Trans. Signal Process., vol. 55, no. 5, pt. 1, pp. 1741-1757, May 2007.

[5] A. Papoulis, "Generalized sampling expansion," IEEE Trans. Circuits Syst., vol. 24, pp. 652-654, 1977.

[6] M. Unser and J. Zerubia, "A generalized sampling theory without band-limiting constraints," IEEE Trans. Circuits Syst. II, Analog Digit. Signal Process., vol. 45, no. 8, pp. 959-969, Aug. 1998.

[7] J. Kusuma and V. K. Goyal, "Multichannel sampling of parametric signals with a successive approximation property," in Proc. IEEE Int. Conf. Image Processing, 2006, pp. 1265-1268.

[8] L. Baboulaz and P. L. Dragotti, "Distributed acquisition and image super-resolution based on continuous moments from samples," in Proc. IEEE Int. Conf. Image Processing, 2006, pp. 3309-3312.

[9] M. Unser and T. Blu, "Cardinal exponential splines: Part I-Theory and filtering algorithms," IEEE Trans. Signal Process., vol. 53, no. 4, pp. 1425-1438, Apr. 2005. 